\title{
Polarimetry with SPI
}

\author{
D. R. Willis ${ }^{1,2} \dagger$, D. J. Clark ${ }^{3}$, R. Diehl ${ }^{1}$, L. Hanlon ${ }^{4}$, G. Kanbach ${ }^{1}$, \\ B. McBreen ${ }^{4}$, S. McGlynn ${ }^{4}$ and A. Strong ${ }^{1}$ \\ ${ }^{1}$ MPE, MPI, Garching, Munich, Germany \\ ${ }^{2}$ ISDC, Chemin D'Ecogia 16, Versoix, CH-1290, Switzerland \\ ${ }^{3}$ School of Physics and Astronomy, University of Southampton, SO17 1BJ, UK \\ ${ }^{4}$ Space Science and Advanced Materials Lab, National University of Ireland, UCD, Dublin 4, \\ Ireland
}

\begin{abstract}
Polarimetry in gamma-rays has the capability to enhance our understanding of compact object emission in our galaxy. In particular this diagnostic method could provide useful insight into the geometrical arrangement of these emitting objects and the roles that magnetic fields play in their emisson mechanisms. Gamma Ray Bursts have been studied in this way but the results, perhaps indicating a high degree of polarisation, remain unverified [Coburn \& Boggs (2003), Wigger et al. (2004), Willis et al. (2005)]. The nature of GRBs solve many instrumental problems in polarimetry, however their true nature is less well defined and so a study of a better understood object such as the Crab Pulsar, for now, may reveal more as to the physics of the system.
\end{abstract}

Keywords. polarization, instrumentation: polarimeters, techniques: polarimetric, gamma rays: observations.

\section{Introduction}

Polar cap models for the Crab Pulsar give gamma-ray emission from pair-production cascades with either curvature radiation or inverse Compton scattered photons from primary particles accelerated in the polar caps of neutron stars, with magnetic photon splitting acting to attenuate in some models. All mechanisms apart form the pairproduction exhibit charactericics of polarisation. The outer gap models involve particles accelerating in charge depletion layers further away from the magnetic poles. And give gamma-ray emission by synchrotron radiation or inverse Compton scattering. Again, clear polarisation signatures can be expected from both mechanisms. Phase resolved and multi-wavelength polarimetric measurements will distinguish between these models.

SPI has 19 hexagonal Germanium detectors, operating in the $20 \mathrm{keV}-8 \mathrm{MeV}$ band. By observing the angular distribution of the multiple events Compton scattered between the detectors and comparing this to the systematic angular distribution of these scattered events, a value for polarisation can be obtained.

\section{Characterising the Scattering Response for Analysis}

Simply histogramming up bins every 60 degrees for a staring observation has been unsuccessful in that any polarisation induced distribution is hidden by the systematic background. To search for this modulation both the background and the systematic structure needs to be removed. The chacterised multiple event response needs to be investigated. To do this a simple dual-detector model is constructed within the GEANT4

$\dagger$ Present address: ISDC, Chemin D'Ecogia 16, Versoix, CH-1290, Switzerland. 


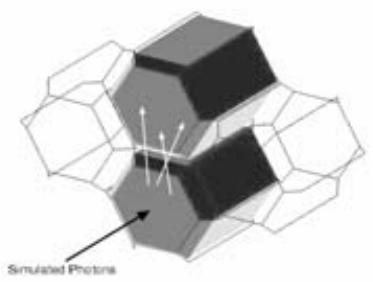

Figure 1. A simple GEANT model of two SPI germanium detector units with two passive units adjacent. Photons are fired into the lower units and the events selected on their interaction with the upper unit.

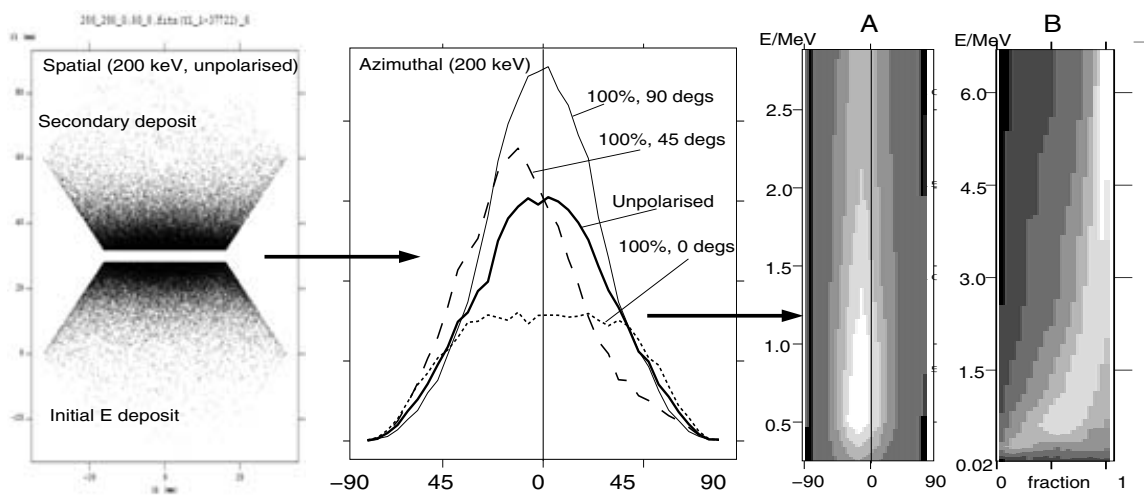

Figure 2. From the simple GEANT model each aspect to the scattering can be quantified. From the spatial distribution on the far left, to the polarisation dependent angular response, to a full energy matrix of the angular response $(\mathbf{A})$. The energy fraction of the energy deposited in the inital detector is also characterised with energy into a matrix (B).

particle/photon simulation software (see Figure 1). Two adjacent detectors are also included as passive attenuators. Mono-energetic flux is fired into one of the detectors and events recorded when a multiple event occurs. Included in the model are simply the Germanium with their respective Aluminium casings. The spatial and so angular distributions can be studied, with respect to energy (see Figure 2).

Using this simple model, a 4-dimensional matrix can be built up dependent on energy, off-axis angle and the percentage and angle of polarisation. This matrix can be employed in one of two ways. Firstly, it can be used to filter all phase-resolved multiple events or used in pattern searching. Secondly, a full set of IRFs (SPI Instrument Response Functions) can be taken and altered for a hypothetical fully polarised flux at 0 and 90 degrees on the detector plane. These two IRFs can then be applied to SPI fitting software, such as SPIMODFIT. By fitting to two separate sources in identical positions, but each using a different polarised IRF, the ratio between the two will give both the degree and angle of polarisation.

\section{References}

Coburn, W. \& Boggs, S. E. 2003, Nature 423, 415

Wigger, C., et al. 2004, ApJ 613, 1088-1100

Willis, D. R., et al. 2005, A\&A A 439, 245-253

Smith, F. G., et al. 1988, MNRAS 233, 305-319 